\title{
Orbit-associated tumors: navigation and control of resection using intraoperative computed tomography
}

\author{
*Nicole A. Terpolilli, MD, ${ }^{1}$ Walter Rachinger, MD, ${ }^{1}$ Mathias Kunz, MD, ${ }^{1}$ Niklas Thon, MD, ${ }^{1}$ \\ Wilhelm H. Flatz, MD, ${ }^{2}$ Jörg-Christian Tonn, MD, ${ }^{1}$ and Christian Schichor, MD1 \\ Departments of ${ }^{1}$ Neurosurgery and ${ }^{2}$ Clinical Radiology, Munich University Medical Center, Munich, Germany
}

\begin{abstract}
OBJECTIVE Treatment of skull base lesions is complex and usually requires a multidisciplinary approach. In meningioma, which is the most common tumor entity in this region, resection is considered to be the most important therapeutic step to avoid tumor recurrence. However, resection of skull base lesions with orbital or optic nerve involvement poses a challenge due to their anatomical structure and their proximity to eloquent areas. Therefore the main goal of surgery should be to achieve the maximum extent of resection while preserving neurological function. In the postoperative course, medical and radiotherapeutic strategies may then be successfully used to treat possible tumor residues. Methods to safely improve the extent of resection in skull base lesions therefore are desirable. The current study reports the authors' experience with the use of intraoperative $\mathrm{CT}$ (iCT) combined with neuronavigation with regard to feasibility and possible benefits of the method.
\end{abstract}

METHODS Those patients with tumorous lesions in relationship to the orbit, sphenoid wing, or cavernous sinus who were surgically treated between October 2008 and December 2013 using iCT-based neuronavigation and in whom an intraoperative scan was obtained for control of resection were included. In all cases a second iCT scan was performed under sterile conditions after completion of navigation-guided microsurgical tumor resection. The surgical strategy was adapted accordingly; if necessary, resection was continued.

RESULTS Twenty-three patients (19 with WHO Grade I meningioma and 4 with other lesions) were included. The most common clinical symptoms were loss of visual acuity and exophthalmus. Intraoperative control of resection by iCT was successfully obtained in all cases. Intraoperative imaging changed the surgical approach in more than half $(52.2 \%)$ of these patients, either because iCT demonstrated unexpected residual tumor masses or because the second scan revealed additional tumor tissue that was not detected in the first scan due to overlay by osseous tumor parts; in these cases resection was continued. In the remaining cases resection was concluded as planned because iCT verified the surgeon's microscopic estimation of tumor resection status. Postoperative visual outcome was favorable in more than $80 \%$ of patients.

CONCLUSIONS Intraoperative CT allows control of resection in case of uncertainty and can help to improve the extent of maximal safe resection, especially in case of osseous tumor parts and masses within the orbit.

http://thejns.org/doi/abs/10.3171/2015.5.JNS15330

KEY WORDS spheno-orbital meningioma; image-guided surgery; intraoperative computed tomography; neuronavigation; skull base; oncology

$\mathrm{S}$ KULL base tumors with close anatomical association to orbit, optic nerve, and the cavernous sinus often cause neurological symptoms like loss of visual function and exophthalmus. In most cases resection (complete or partial removal) is the most promising and most effective therapeutic approach with regard to preservation or restoration of visual function. Furthermore, operative tumor removal or reduction is a crucial step in multidisciplinary operative as well as medical or radiotherapeutic treatment concepts, because it allows exact histological diagnosis and safe decompression of anatomical structures at risk. However, resection of these tumors is associated with increased perioperative morbidity due to the proximity of highly sensitive structures-e.g., cranial nerves

ABBREVIATIONS CN = cranial nerve; iCT = intraoperative CT; iMRI = intraoperative MRI; NNS = neuronavigational system.

SUBMITTED February 11, 2015. ACCEPTED May 14, 2015.

INCLUDE WHEN CITING Published online November 13, 2015; DOI: 10.3171/2015.5.JNS15330.

* Drs. Terpolilli and Rachinger contributed equally to this work. 
(CNs) II, III, IV, and VI; the carotid artery; and the cavernous sinus. Furthermore, osseous tumor parts typically grossly distort normal anatomy, which further complicates surgical removal.

In an effort to improve resection safety, the use of image-guided surgery via various neuronavigational systems (NNSs) has been proposed. In the last few years its successful application during resection of orbit-associated tumors has been anecdotally reported. . $^{10,11,13,17,29,34}$ The existing literature suggests that neuronavigation is a helpful and valuable tool to reduce perioperative morbidity while improving resection grade. However, the cases described in the literature used preoperatively obtained imaging (CT, MRI, or a combination of both) for the navigation. Intraoperative CT (iCT) obtained by a multislice scanner that is mounted in the operation theater allows for quick performance of scans that can be used for referencing of the NNS after the patient has been positioned for the operation. The combination of $\mathrm{iCT}$ and NNS has been proven to be highly accurate and surgically precise in other parts of the cranium. ${ }^{32}$ In our experience so far, $, 33,35$ iCT provides excellent NNS accuracy and the possibility to perform further intraoperative scans to update NNS data. Even more importantly, iCT scanning offers the possibility of intraoperative imaging of spheno-orbital tumors as a control of resection. iCT scanning, especially intraoperative control of resection by iCT, may therefore be used to increase the extent of resection in cases of uncertainty while providing maximum navigation accuracy and, thereby, safety for critical anatomical structures.

The aim of this study was to evaluate iCT-guided resection of orbit-associated tumors and iCT control of resection with regard to feasibility and possible benefits of the method.

\section{Methods \\ Patient Population}

Patients undergoing microsurgical resection of skull base or orbit-associated lesions between October 2008 and December 2013 using CT-guided neuronavigation were screened. Patients gave informed consent for the operation and the use of $\mathrm{iCT}$ navigation, including performance of the baseline CT scan and possible further intraoperative scans for control of resection. Only those patients who received a second intraoperative scan were included in the final analysis.

\section{Preoperative Imaging for Navigation}

The standard image modality for image-guided surgery is cranial MRI; therefore contrast-enhanced, highresolution T1- and T2-weighted imaging in 3 planes was performed on the day prior to surgery when possible. A DOTATOC-PET scan was obtained in cases of extensive and/or recurrent meningioma to evaluate the extent to which osseous structures were affected by the tumor, especially in cases in which PET scanning had been previously performed for follow-up or in cases where postoperative radiotherapy was planned or discussed. As recently demonstrated, ${ }^{26}$ DOTATOC-PET helps to discern normal brain parenchyma and tumor tissue intraoperatively, and so this modality was implemented into navigation when available.

\section{Neuronavigation and iCT}

The intraoperative setup has been described previously. ${ }^{33,35}$ In short, preoperatively obtained imaging was fused using the navigation software. A ceiling-mounted frameless infrared-based NNS (Vector Vision, BrainLab) was used during the operation. After positioning the patient for surgery on the carbon operating table using a radiotranslucent Mayfield clamp made of carbon with radiolucent skull pins (Integra), baseline imaging was obtained with a 40-multislice sliding-gantry CT scanner (Somatom Sensation Open Sliding Gantry, Siemens Medical Solutions) with collimation at $120 \mathrm{kV}, 320 \mathrm{mAs}$, a pitch of 0.9 , and a rotation time of 1 second. Images were then reconstructed in $3 \mathrm{D}$ in 0.75 - to $1.0-\mathrm{mm}$ slice thickness. The CT scanner was directly connected to the NNS, allowing direct transfer of the data for automated registration; the registration accuracy was then ascertained by touching 6 superficial anatomical landmarks (e.g., acoustic meatus) with a nonsterile indicator tool. If registration accuracy was calculated to be deviated by more than $1.5 \mathrm{~mm}$, the coregistration process was repeated. If accuracy was deemed sufficient, the gantry was moved into parking position. After skin sterilization and sterile draping microsurgical tumor resection was performed according to the department standard by using a previously established work flow for CTnavigated cranial operations. ${ }^{33}$

After the preoperatively established goal of the operation (complete tumor resection, partial resection of critical tumor parts, optic nerve decompression) was achieved as judged by the responsible surgeon, an intraoperative second CT scan was performed when deemed necessary (in cases of complex and/or distorted anatomy, recurrent tumor). For this purpose sterile draping was applied after removal of all metal objects in the operation site, and the CT scan was repeated using the same parameters as the baseline scan.

\section{Data Analysis}

Data collection and analysis was performed retrospectively using patient files and radiographic data obtained pre-, intra-, and postoperatively.

\section{Results \\ Patients Characteristics}

Fifty-two patients with orbit-associated lesions received iCT-guided neuronavigation during the recruitment period. In 23 cases a second scan was requisitioned by the surgeon. These 23 patients, 21 (91.3\%) of whom were female and $2(8.7 \%)$ of whom were male (Table 1), were included in this retrospective analysis. The mean age at operation was $49 \pm 10$ years (mean \pm SD). Nine of $23(39 \%)$ patients presented to our department with recurrent tumor growth and had undergone at least 1 previous operation.

The most common symptoms on admission to the hospital were loss of visual acuity and/or visual field defects $(10 / 23,43.5 \%)$, exophthalmus $(13 / 23,56.5 \%)$, and double vision $(10 / 23,43.5 \%)$. However, only 2 patients had had 


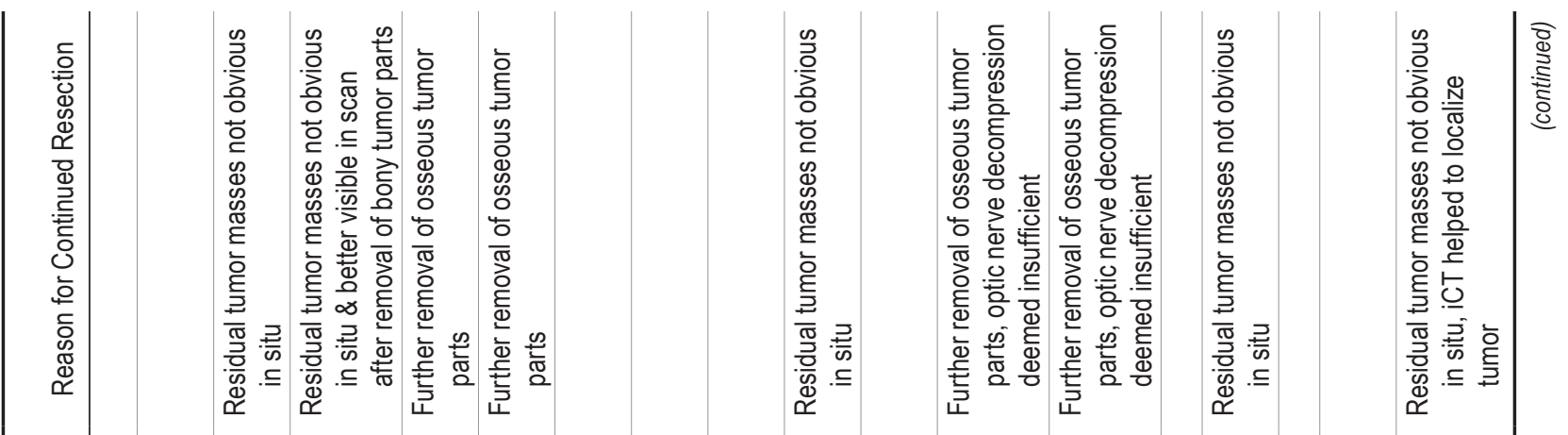

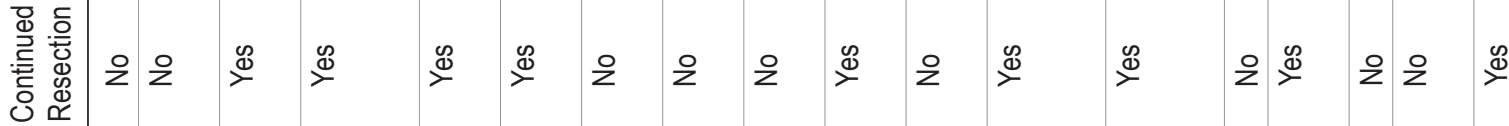
는

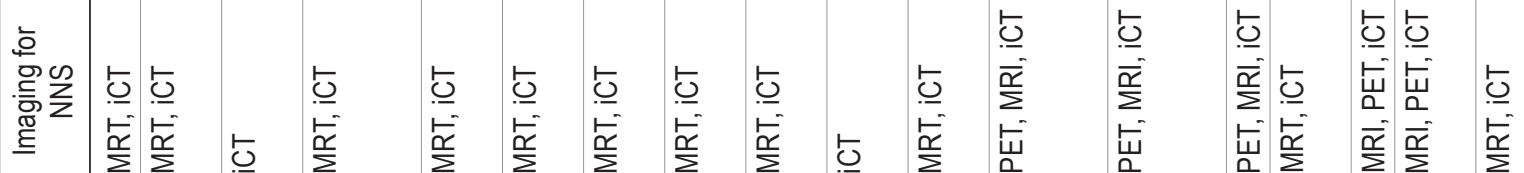

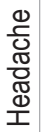

$\frac{0}{\infty}$

呆

$\ddot{x}$

$\exists \quad \exists \quad \exists \quad \vec{x} \quad \vec{x} \quad \vec{x} \quad \vec{x} \quad \vec{x}$

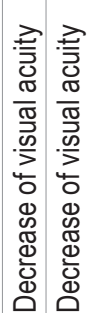

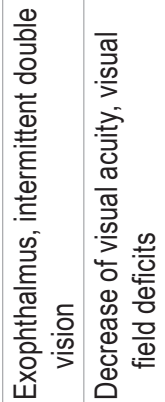

듷ำ

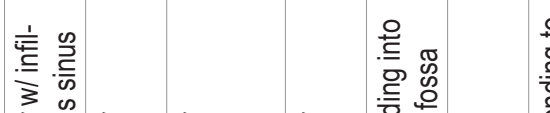

宽

秃

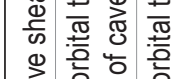

旁

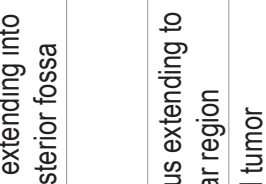

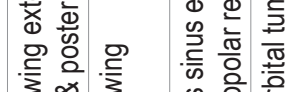

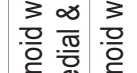

응 잉 은

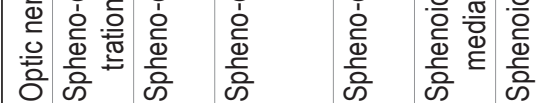

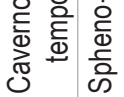

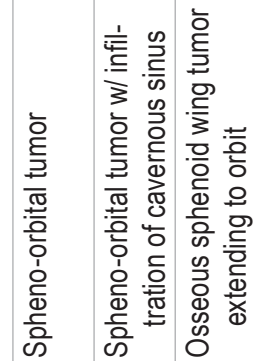

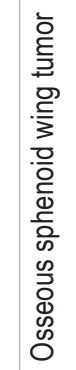

$\exists \mathbb{x}$

$\vec{\square} \vec{\square} \vec{\square}$

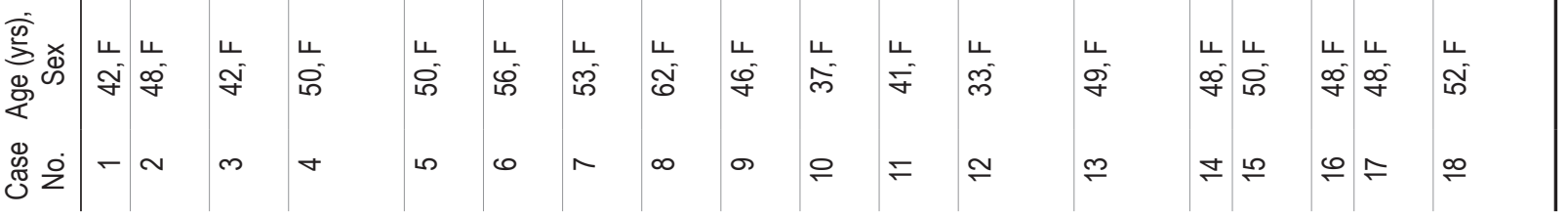




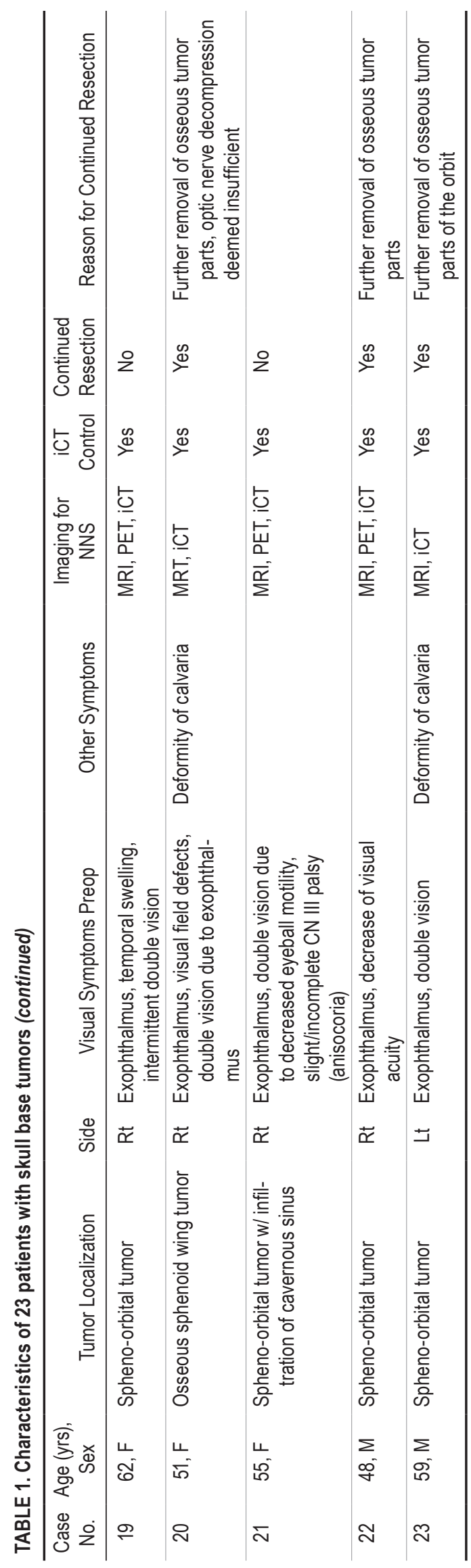

a manifest eye muscle paresis (CN VI, $1 / 23,4.3 \%$; $\mathrm{CN}$ III and IV, 1/23, 4.3\%); in the other cases eye movements were restricted because of proptosis. Four patients in total displayed $\mathrm{CN}$ lesions. Other frequent complaints included headache and deformities of the calvaria or cosmetic disturbances. The majority of tumors were localized in the region of the sphenoid wing, with invasion of the orbit (14 patients, $60.9 \%$ ) or without invasion of the orbit but inducing compression of the optic nerve/optic canal (6 patients, $26.1 \%)$. In 3 cases (13\%) the lesion was confined to the orbit or the optic nerve/optic canal.

\section{Neuronavigation and Baseline iCT Scan}

Neuronavigation was successfully used in all cases; in 21/23 patients (91.3\%) baseline CT data were fused with preoperatively obtained MR images (T1, T1 with contrast, T2), and in 5 cases $(21.7 \%)$ neuronavigation was performed based on preoperative MR images fused with DOTATOC-PET-CT. In the 2 remaining cases (8.7\%), in which MRI could not be performed due to claustrophobia and a non-MRI-compatible pacemaker, we used CT data only. Figure 1 shows representative NNS screen shots obtained while using MRI-iCT-based (Fig. 1 left) or PETiCT-based (Fig. 1 right) data. Baseline scanning and the referencing of the NNS was performed within 15 minutes in all cases, and radiation exposure was $62.7 \pm 3.2 \mathrm{mGy}$ (mean $\pm \mathrm{SD})$.

\section{Operation and iCT Control}

No aggressive effort at complete tumor removal was attempted in the region of the cavernous sinus when there was tumor infiltration $(n=5)$. Tumor-affected bone was removed when possible, or drilled off using a diamond-tip high-speed drill. A second iCT scan was performed under sterile conditions to ascertain tumor resection status; the expenditure of time necessary was less than 15 minutes, and the exposure dose was $63.7 \pm 3.7 \mathrm{mGy}($ mean $\pm \mathrm{SD})$. The images were reconstructed in the axial plane in 1-mm sections in bone and tissue kernel, and then the second iCT scan was imported into the neuronavigation system and fused with the preoperative iCT. Image quality was excellent as far as osseous structures were concerned, and it was good for soft-tissue imaging, all intraoperative control CTs were suitable for image fusion. There were some artifacts in the region of the head pins; because the pins were placed outside of the region of interest (skull base, orbit) these were negligible as far as navigation was concerned. After craniotomy the air surface artifacts were increased, deteriorating the image quality for soft tissue (see Figs. 2 and 3, panel C, lower row). However, the image quality for osseous structures was good (see Figs. 2 and 3, panel C, upper row), so NNS viability and accuracy was not impaired.

Navigation accuracy was then reconfirmed, and brain shift was negligible in the regions of interest at the skull base. Artifacts were observed at the air-brain surface, and these impaired the quality and resolution of the iCT scan in the superficial temporal region. However, skull base and skull base structures could be assessed without a problem, especially in bone kernel reconstructions. 

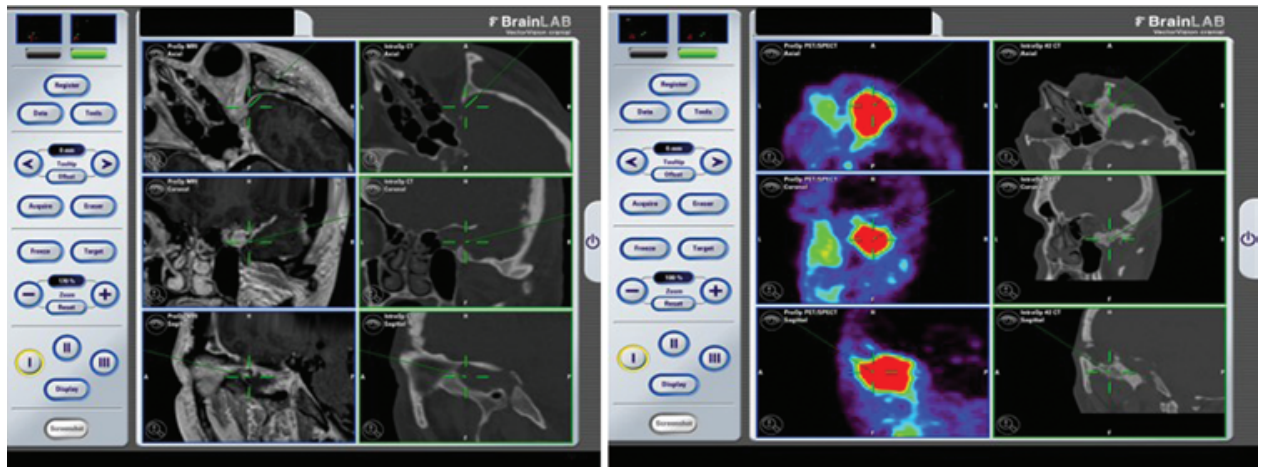

FIG. 1. Intraoperative neuronavigation screen shots. Left: Preoperative T2-weighted MRI overlaid with baseline iCT. Right: Preoperative DOTATOC-PET overlaid with baseline iCT. Figure is available in color online only.

Figures 2-4 show illustrative cases; pre- and postoperative MRI is represented on the left and right side, respectively. In the middle of the panels the baseline iCT and iCT resection control scans are shown. After evaluation of the scan, tumor resection was concluded in $11(47.8 \%)$ cases because iCT confirmed achievement of the intended operative result. However, in the other 12 cases resection was continued: in 6 cases, CT scanning revealed tumor masses intended for resection that were not obvious in situ (50\%), in 3 patients the second scan revealed tumor tissue not obvious in the baseline examination due to overlay by bony tumor sections (25\%). Intraoperative resection control proved to be especially helpful in evaluating extent of resection of osseous tumor parts: in 5 of the 12 patients $(41.7 \%)$ resection was extended because optic nerve de- compression was deemed insufficient radiomorphologically. Figure 5 shows 2 examples (Cases 12 and 22). In 1 patient (Case 15), the tumor was situated intraorbitally and was hard to localize intraoperatively; therefore we performed iCT after insertion of a cotton pad immersed in contrast agent, which helped to localize the tumor and define its margins. In 3 cases where contrast was applied, the position and placement of orbital reconstruction material could be assessed, and was adapted in 1 case.

\section{Postoperative Outcome}

Histopathological examination showed meningioma WHO Grade I in the majority (19 [82.6\%]) of patients. In the remaining cases the pathological diagnosis was chon-
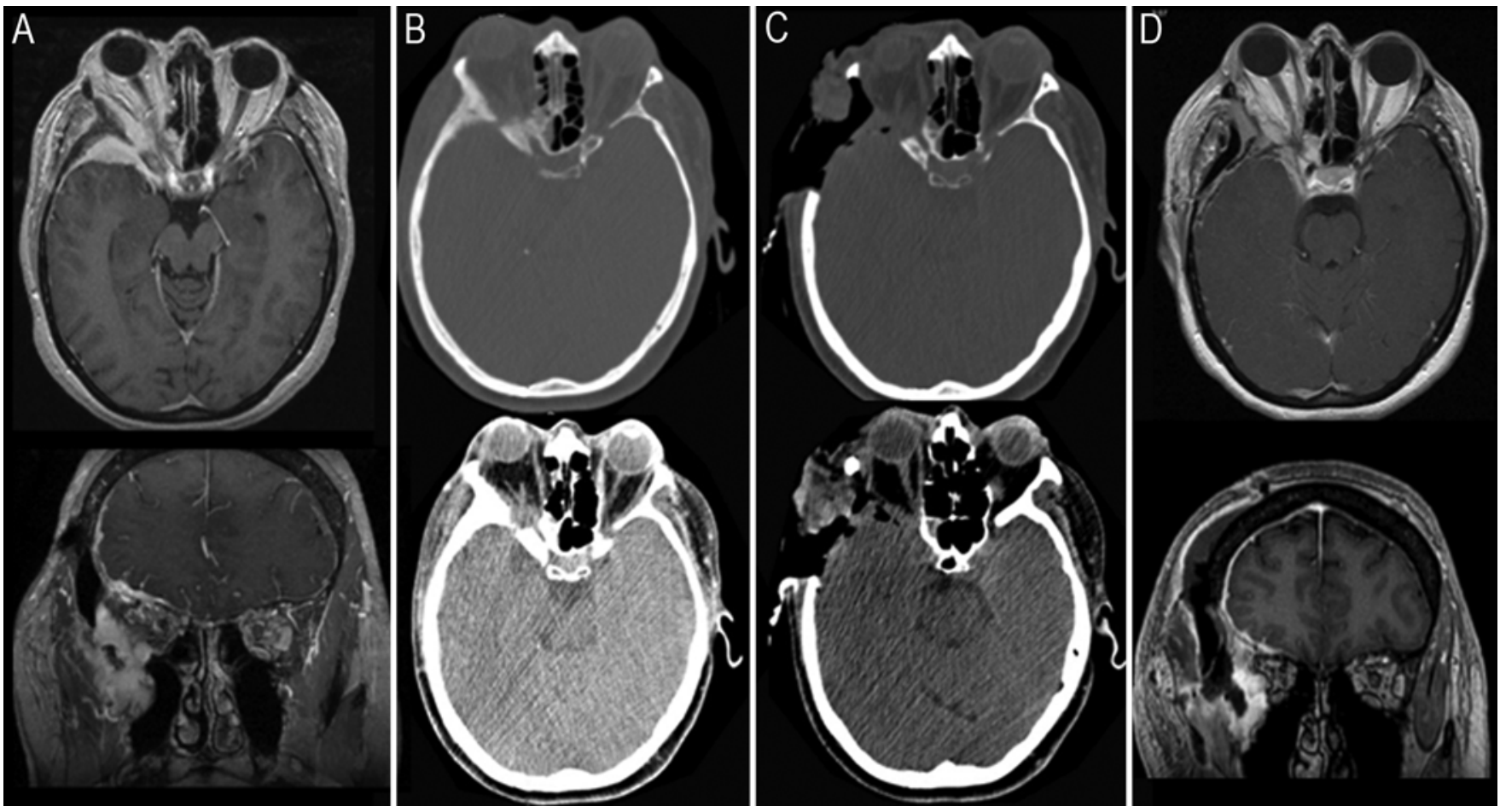

FIG. 2. Case 16. This patient presented with headache, and histological findings were of meningioma WHO Grade I (A). Intraoperative $\mathrm{CT}$ scans were obtained before $(\mathrm{B})$ and after $(\mathrm{C})$ resection. Because the second iCT scan demonstrated no residual tumor masses impacting the orbit and good osseous decompression of the orbit, resection was concluded. Postoperative MRI study (D) showed no residual tumor masses. 

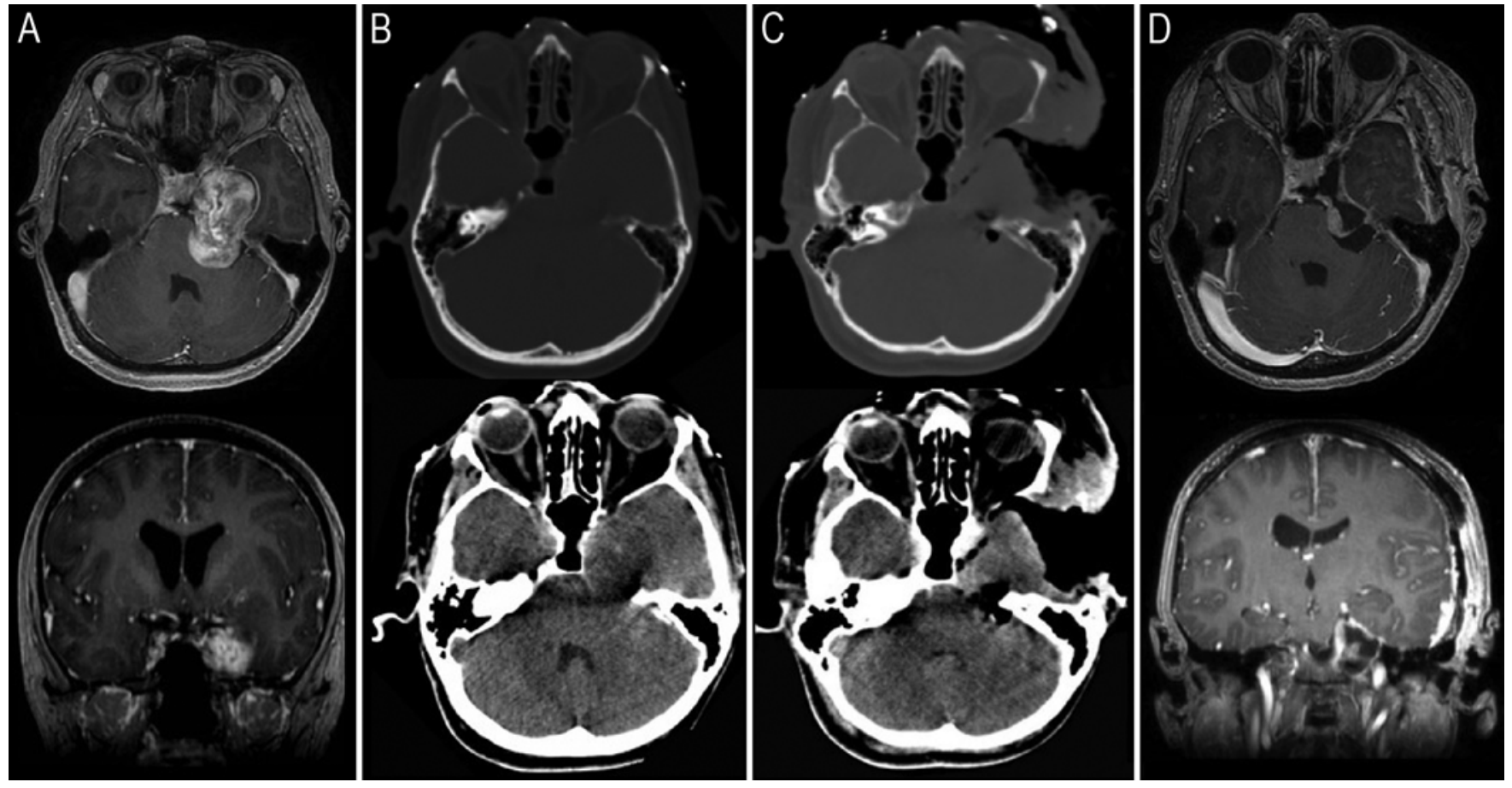

FIG. 3. Case 6. This patient presented with blurred vision and symptomatic epileptic seizures. MRI obtained before operation (A). Initial iCT scan (B). The second iCT scan (C) revealed additional tumor masses, so resection was continued. Postoperative MRI sequence (D) displayed efficient tumor removal (as far as possible from the [temporal] approach chosen) and good decompression of the orbit.

drosarcoma WHO Grade II (1 [4.3\%]), sarcoma (2 [8.7\%]), and neurinoma WHO Grade I (1 [4.3\%]). No new CN deficits or oculomotor deficits were observed. Two patients developed subgaleal CSF collection that required intervention.

Three months after operation, visual acuity and/or visual field defects remained stable or improved in $82.6 \%$ of patients. Exophthalmus was resolved or significantly reduced within 3 months of operation in 11/13 patients initially presenting with this symptom. Within this series, tumor recurrence requiring operation has occurred in only 2/19 patients with meningioma WHO Grade I so far (4.5 and 4.9 years after the initial operation).

\section{Discussion}

Tumors of the skull base, especially sphenoid ridge or spheno-orbital meningiomas, have a complex anatomy and involve cranial nerves, major blood vessels, and the optical system including the orbit. Because skull base tumors often have extended osseous components that grossly distort normal anatomy, intraoperative orientation might be further compromised. . 16,25 Therefore, aggressive resection can be associated with significant morbidity, and previously many of these lesions were considered unresectable. The development of complex neurosurgical approaches to the skull base and orbit as well as interdisciplinary operation teams including ophthalmic, otorhinolaryngological, and craniomaxillofacial surgeons led to a drop in morbidity rates while extent of resection increased. ${ }^{12,27,28,31}$ However, even in these series, postoperative morbidity was reported to occur at least transiently in $30 \%{ }^{27}$ to $69 \%$ of patients. ${ }^{28}$
Because extended skull base tumors involving bone, dura mater, and the orbital system are hard to adequately resect, recurrence rates are high. Although 5- to 10-year regrowth rates of $50 \%$ and more ${ }^{1,9,21}$ seem to be a thing of the past, complete resection is seldom achieved. ${ }^{6,20,27}$ However, there is a clear correlation between extent of resection and recurrence rate. ${ }^{18-20}$ Furthermore, surgical debulking of tumor masses is often necessary to achieve an exact histological diagnosis, and decompression of neurological structures at risk is a prerequisite for subsequent radiation therapy or radiosurgery. Maximum possible resection should be achieved while reducing the risk of new deficits. Several groups reported the successful use of various neuronavigation devices based on preoperatively acquired $\mathrm{CT}$ or MRI scans during surgical removal of orbit-associated tumors. $37,8,11,13,15,34$ Most researchers conclude that the use of image-guided surgery is especially helpful in planning the optimal surgical approach and increasing resection grade independently of the tumor entity ${ }^{3,15,29}$ while increasing safety, thus reducing if not completely avoiding operative morbidity.

In this study we combined an NNS with iCT for resection of orbit-associated tumors. iCT-based neuronavigation is advantageous because it allows referencing to be done in the same position in which the surgery is performed; thus navigation accuracy is improved by eliminating position-dependent brain shift. As previously demonstrated, $\mathrm{iCT}$ can be integrated into the operative work flow without major delay of the operation and major changes to the operative routine. ${ }^{33,35}$ The possibility of performing intraoperative imaging allows for control of resection and updating of the NNS data. In more than half of the cases this 

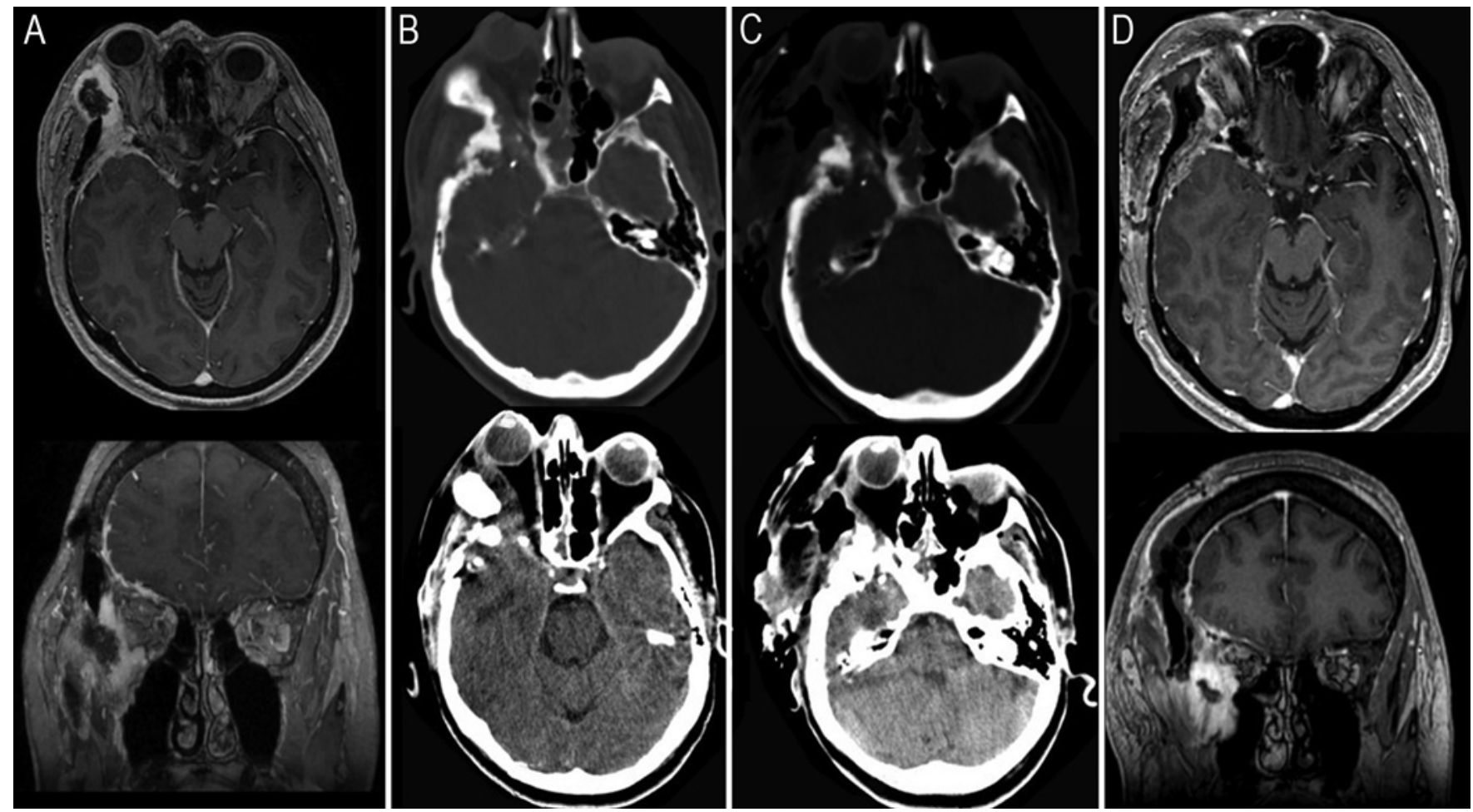

FIG. 4. Case 20. This patient presented with exopthalmus and impaired eye motility due to recurrent spheno-orbital meningioma (WHO Grade I). Preoperative MRI (A). Baseline iCT (B). Intraoperative CT (C) revealed additional tumor masses, and resection was continued. Postoperative MRI (D) showed sufficient optic nerve decompression. Radiation therapy was initiated postoperatively.

information led to continuation of tumor removal, which would have been terminated otherwise. In this retrospective series we did not experience adverse outcome; on the contrary, visual function was conserved or improved in the vast majority of cases. It can therefore be considered a most valuable tool for safely maximizing the extent of tumor resection and can add valuable information, especially in cases in which orientation is hindered.

One major drawback of iCT, however, is exposure to radiation. A number of groups therefore favor intraoperative MRI (iMRI) in combination with neuronavigation. ${ }^{5,23,24}$ iMRI has quickly been proven to be very valuable in glioma surgery; due to its superior resolution of brain parenchyma and glioma tissue, iMRI has been shown to improve the extent of resection in low-grade as well as high-grade gliomas. ${ }^{4,14,30}$ Furthermore, iMRI resection control imaging is advantageous due to the absence of exposure to x-rays. However, iMRI is quite time consuming, requires a dedicated operating theater and operating room equipment, and is significantly more costly than CT. In the context of spheno-orbital tumors, iMRI has the additional drawback that the commonly existing bony tumor parts are not ideally imaged; because osseous fractions might constitute the main bulk of spheno-orbital and/or skull base tumors, iMRI most probably is not as helpful as iCT as far as the intraoperative control of resection is concerned.

Furthermore, patients with spheno-orbital tumors usually undergo pre- and postoperative CT scanning even if they receive MRI and PET scans to evaluate bony tumor sections; in our series, preoperative CT scanning was not performed in cases in which $\mathrm{iCT}$ was planned. We therefore think that in the special case of orbit-associated tumor resection, iCT might be superior to iMRI as far as intraoperative imaging and intraoperative control of resection is concerned.

This is the first study to introduce iCT-based neuronavigation to skull base surgery. In our experience, the main advantages of iCT examinations were good depiction of osseous tumor residues, high neuronavigation accuracy due to little brain shift in the region of the skull base, and short examination duration allowing for repeated assessments without undue delay of the operation. Routine application of iCT-based neuronavigation during skull base tumor surgery therefore is feasible and expedient. In more than half of the patients tumor removal was extended after the intraoperative control of resection, indicating that iCT significantly influenced extent of resection. In the present series the neurological and ophthalmological outcome was favorable, and in more than $80 \%$ of patients visual acuity was stable or improved. There were no perioperative deaths. Compared with other large series of skull base tumor resection ${ }^{9,17-19,22,27}$ the complication rate was low. So far, recurrence requiring operation occurred in only 2 patients. Given the small number of patients the study inevitably has limitations. Whereas we do not propose intraoperative control of resection as a standard procedure for all skull base operations, it can be very helpful in a highly selected cohort of patients in whom complex tumor configuration and distorted anatomy due to previous operation(s) and/or tumor growth impede exact appraisal of resection status. In these cases it may lead to more ag- 

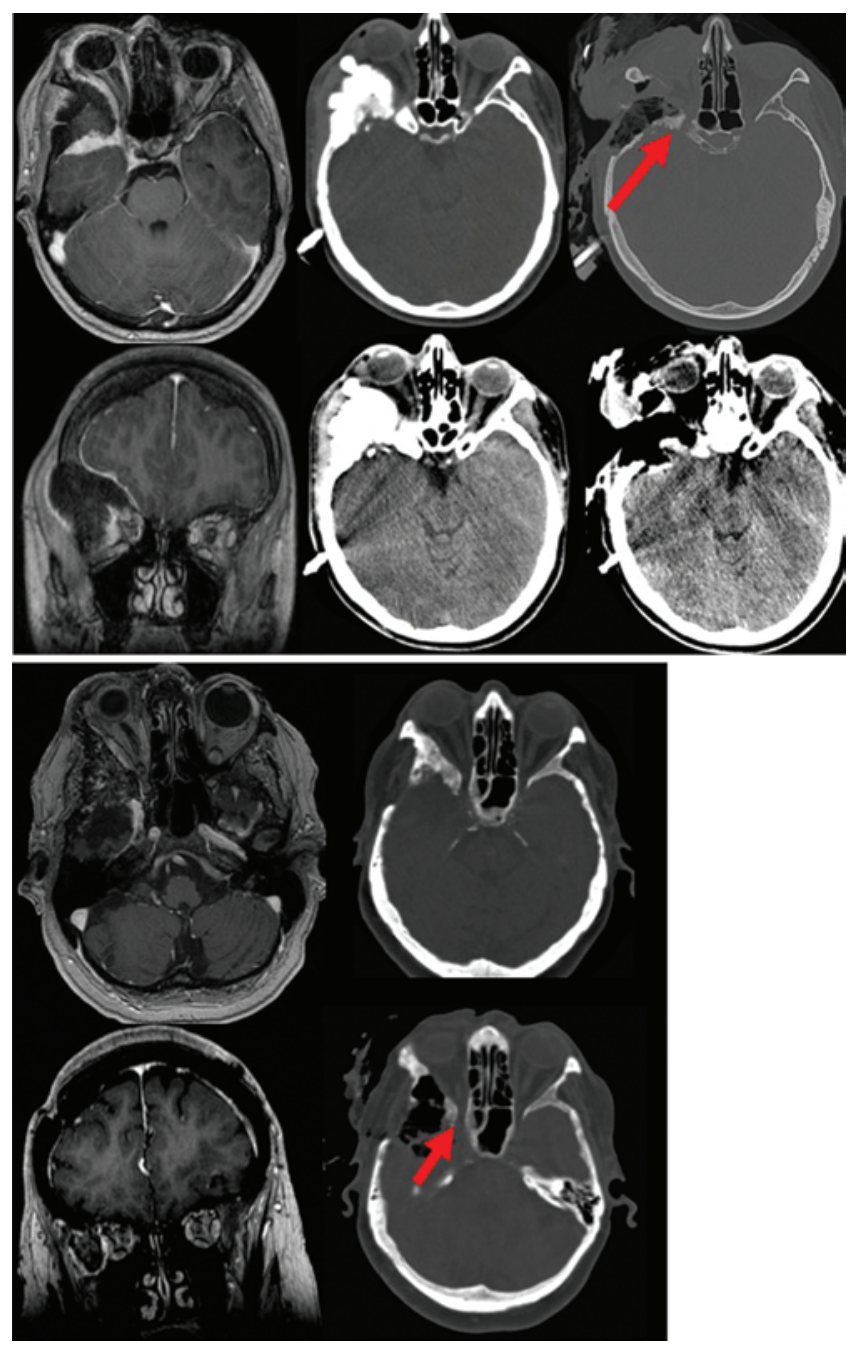

FIG. 5. iCT was most helpful in the appraisal of osseous tumor parts and their resection status. Upper Rows: Case 12. This patient presented with exopthalmus and skull deformity. The preoperative MRI (left) showed extensive osteomeningioma. The initial iCT scan (center) used for navigation confirms an extensive osseous tumor component impacting the orbit and optic canal. The second iCT (right) showed insufficient osseous decompression of the orbit (arrow), and resection was therefore continued. Lower Rows: Case 22. In this recurrent osteomeningioma radiation therapy was planned after decompression of the optic nerve; the second iCT scan (lower right) revealed incomplete optic nerve decompression (arrow), so resection was continued. Figure is available in color online only.

gressive yet safe tumor removal, and thus to a better outcome. Timing of the intraoperative scan and experience of the surgeon may influence the usefulness of the information gained by $\mathrm{iCT}$.

\section{Conclusions}

Further investigation is needed to determine whether intraoperative control of resection by iCT significantly improves outcome and lowers recurrence rates. However, our feasibility study indicates that iCT-based navigation with intraoperative resection control may be a valuable tool in orbit-associated and skull base tumor surgery.

\section{References}

1. Adegbite AB, Khan MI, Paine KW, Tan LK: The recurrence of intracranial meningiomas after surgical treatment. J Neurosurg 58:51-56, 1983

2. Behari S, Giri PJ, Shukla D, Jain VK, Banerji D: Surgical strategies for giant medial sphenoid wing meningiomas: a new scoring system for predicting extent of resection. Acta Neurochir (Wien) 150:865-877, 2008

3. Berger M, Char DH: Interactive image guidance for surgical localization of orbital apical tumors. Orbit 21:199-203, 2002

4. Black PM, Alexander E III, Martin C, Moriarty T, Nabavi A, Wong TZ, et al: Craniotomy for tumor treatment in an intraoperative magnetic resonance imaging unit. Neurosurgery 45:423-433, 1999

5. Black PM, Moriarty T, Alexander E III, Stieg P, Woodard EJ, Gleason PL, et al: Development and implementation of intraoperative magnetic resonance imaging and its neurosurgical applications. Neurosurgery 41:831-845, 1997

6. Bonnal J, Thibaut A, Brotchi J, Born J: Invading meningiomas of the sphenoid ridge. J Neurosurg 53:587-599, 1980

7. Camara JG, Nguyen LT, Fernandez-Suntay JP, Nardin GF, Sua AS: The use of a computer-assisted image-guided system (InstaTrak) in orbital surgery. Ophthal Plast Reconstr Surg 17:447-451, 2001

8. Cascone P, Santamaria S, Mercurio A, Polito E: The use of the neuronavigator in the orbital surgery of a rare case of Rosai-Dorfman disease. J Craniofac Surg 15:651-654, 2004

9. Cophignon J, Lucena J, Clay C, Marchac D: Limits to radical treatment of spheno-orbital meningiomas. Acta Neurochir Suppl (Wien) 28:375-380, 1979

10. Enchev Y, Tzekov C, Ferdinandov D, Cekov A, Spiriev T: Neuronavigation in cranioorbital neurosurgery - do we really need it? Turk Neurosurg 21:119-126, 2011

11. Hejazi N: Frameless image-guided neuronavigation in orbital surgery: practical applications. Neurosurg Rev 29:118-122, 2006

12. Honeybul S, Neil-Dwyer G, Lang DA, Evans BT, Ellison DW: Sphenoid wing meningioma en plaque: a clinical review. Acta Neurochir (Wien) 143:749-758, 2001

13. Karcioglu ZA, Mascott CR: Computer-assisted image-guided orbit surgery. Eur J Ophthalmol 16:446-452, 2006

14. Kubben PL, ter Meulen KJ, Schijns OE, ter Laak-Poort MP, van Overbeeke JJ, van Santbrink H: Intraoperative MRIguided resection of glioblastoma multiforme: a systematic review. Lancet Oncol 12:1062-1070, 2011

15. Lee KY, Ang BT, Ng I, Looi A: Stereotaxy for surgical navigation in orbital surgery. Ophthal Plast Reconstr Surg 25:300-302, 2009

16. Little KM, Friedman AH, Sampson JH, Wanibuchi M, Fukushima T: Surgical management of petroclival meningiomas: defining resection goals based on risk of neurological morbidity and tumor recurrence rates in 137 patients. Neurosurgery 56:546-559, 2005

17. Marcus H, Schwindack C, Santarius T, Mannion R, Kirollos $\mathrm{R}$ : Image-guided resection of spheno-orbital skull-base meningiomas with predominant intraosseous component. Acta Neurochir (Wien) 155:981-988, 2013

18. Mariniello G, Bonavolontà G, Tranfa F, Maiuri F: Management of the optic canal invasion and visual outcome in spheno-orbital meningiomas. Clin Neurol Neurosurg 115:1615-1620, 2013

19. Mariniello G, Maiuri F, Strianese D, Donzelli R, Iuliano A, Tranfa F, et al: Spheno-orbital meningiomas: surgical approaches and outcome according to the intraorbital tumor extent. Zentralbl Neurochir 69:175-181, 2008

20. Mathiesen T, Lindquist C, Kihlström L, Karlsson B: Recurrence of cranial base meningiomas. Neurosurgery 39:2-9, 1996 
21. Mirimanoff RO, Dosoretz DE, Linggood RM, Ojemann RG, Martuza RL: Meningioma: analysis of recurrence and progression following neurosurgical resection. J Neurosurg 62:18-24, 1985

22. Nakamura M, Roser F, Jacobs C, Vorkapic P, Samii M: Medial sphenoid wing meningiomas: clinical outcome and recurrence rate. Neurosurgery 58:626-639, 2006

23. Nimsky C, Ganslandt O, Buchfelder M, Fahlbusch R: Intraoperative visualization for resection of gliomas: the role of functional neuronavigation and intraoperative $1.5 \mathrm{~T}$ MRI. Neurol Res 28:482-487, 2006

24. Nimsky C, Ganslandt O, Hastreiter P, Wang R, Benner T, Sorensen AG, et al: Preoperative and intraoperative diffusion tensor imaging-based fiber tracking in glioma surgery. Neurosurgery 61 (1 Suppl):178-186, 2007

25. Pieper DR, Al-Mefty O: Management of intracranial meningiomas secondarily involving the infratemporal fossa: radiographic characteristics, pattern of tumor invasion, and surgical implications. Neurosurgery 45:231-238, 1999

26. Rachinger W, Stoecklein VM, Terpolilli NA, Haug AR, Ertl L, Pöschl J, et al: Increased 68Ga-DOTATATE uptake in PET imaging discriminates meningioma and tumor-free tissue. J Nucl Med 56:347-353, 2015

27. Ringel F, Cedzich C, Schramm J: Microsurgical technique and results of a series of 63 spheno-orbital meningiomas. Neurosurgery 60 (4 Suppl 2):214-222, 2007

28. Sandalcioglu IE, Gasser T, Mohr C, Stolke D, Wiedemayer $\mathrm{H}$ : Spheno-orbital meningiomas: interdisciplinary surgical approach, resectability and long-term results. J Craniomaxillofac Surg 33:260-266, 2005

29. Selva D, Chen C, Wormald PJ: Frontoethmoidal osteoma: a stereotactic-assisted sino-orbital approach. Ophthal Plast Reconstr Surg 19:237-238, 2003

30. Senft C, Bink A, Franz K, Vatter H, Gasser T, Seifert V: Intraoperative MRI guidance and extent of resection in glioma surgery: a randomised, controlled trial. Lancet Oncol 12:997-1003, 2011

31. Shrivastava RK, Sen C, Costantino PD, Della Rocca R: Sphenoorbital meningiomas: surgical limitations and les- sons learned in their long-term management. J Neurosurg 103:491-497, 2005

32. Stelter K, Ledderose G, Hempel JM, Morhard DF, Flatz W, Krause E, et al: Image guided navigation by intraoperative CT scan for cochlear implantation. Comput Aided Surg 17:153-160, 2012

33. Uhl E, Zausinger S, Morhard D, Heigl T, Scheder B, Rachinger $\mathrm{W}$, et al: Intraoperative computed tomography with integrated navigation system in a multidisciplinary operating suite. Neurosurgery 64 (5 Suppl 2):231-240, 2009

34. Westendorff C, Kaminsky J, Ernemann U, Reinert S, Hoffmann J: Image-guided sphenoid wing meningioma resection and simultaneous computer-assisted cranio-orbital reconstruction: technical case report. Neurosurgery 60 (2 Suppl 1):E173-E174, 2007

35. Zausinger S, Scheder B, Uhl E, Heigl T, Morhard D, Tonn JC: Intraoperative computed tomography with integrated navigation system in spinal stabilizations. Spine (Phila Pa 1976) 34:2919-2926, 2009

\section{Disclosures}

Dr. Tonn received support for a non-study-related clinical or research effort from BrainLab, and he received speaker's honoraria from BrainLab and Siemens.

\section{Author Contributions}

Conception and design: Schichor, Terpolilli, Tonn. Acquisition of data: all authors. Analysis and interpretation of data: Schichor, Terpolilli, Thon, Flatz, Tonn. Drafting the article: Terpolilli. Critically revising the article: Schichor, Rachinger, Kunz, Thon, Flatz.

\section{Correspondence}

Christian Schichor, Department of Neurosurgery, Munich University Medical Center, Marchioninistr. 15, 81377 Munich, Germany.email: schichor@web.de. 